ous. The above statement is equivalent to the fact that $\lambda(\mathfrak{D})$ $=\mathfrak{O}\left(x^{(i)}\right) \cap \mathfrak{O}\left(x^{(2)}\right) \cap \cdots \cap \mathfrak{}\left(x^{(n)}\right)$, an open set in $S$. Thus $\lambda^{-1}$ is contipuous.

Conversely, for $x \in B^{d}$, let 2 be that subset of $T$ for which $\lambda(Q)$ $=\mathfrak{D}(x)$. Choose $\alpha \in \mathrm{A}$ such that $x=\left\{\left(\alpha, x_{\alpha}\right)\right\}$. Then $y=\left\{M_{\alpha}\right\} \in \mathcal{Q}$ if, and only if, $x_{\alpha} \in M_{\alpha}$. Hence 2 is the relativization of a Cartesian product of factors $\Omega_{\beta}$ if $\beta \neq \alpha$, and $\supseteq\left(x_{\alpha}\right) .2$ is thus open, and $\lambda$ is continuous. This proves that $\lambda$ is indeed a homeomorphism.

\title{
BIBLIOGRAPHY
}

1. G. Birkhoff, Lattice theory, Amer. Math. Soc. Colloquium Publications, vol. 25, rev. ed., New York, 1949.

2. S. Lefschetz, Algebraic topology, Amer. Math. Soc. Colloquium Publications, vol. 27, New York, 1942.

3. R. Sikorski, On the representation of Boolean algebras as fields of sets, Fund. Math. vol. 35 (1948) pp. 247-258.

4. M. H. Stone, Applications of the theory of Boolean rings to general topology, Trans. Amer. Math. Soc. vol. 41 (1937) pp. 375-481.

Washington University in St. Louis

\section{INTEGRAL BASES AND VARIETIES MULTIPLY OF THE FIRST SPECIES}

H. T. MUHLY

1. The object of this note is to point out the connection between varieties multiply of the first species in the sense of Dubreil ${ }^{1}$ and those irreducible varieties $V$ which are such that the ring of homogeneous coordinates of the general point of $V$ admits an independent linear base over the subring generated by a suitably chosen set of independent variables.

2. Let $k$ be a field of characteristic zero and let $R$ $=k\left[X_{0}, X_{1}, \cdots, X_{n}\right]$ be the ring of polynomials in $n+1$ indeterminates over $k$. If $\mathfrak{a}$ is an $H$-ideal in $R$ with no irrelevant components, $\mathfrak{a}$ is said to be of the first species if there exists a linear form $L=\sum c_{i} X_{i}$, $c_{i} \in k$, which does not belong to any of the relevant prime ideals of $\mathfrak{a}$ and is such that $a+R \cdot L$ has no irrelevant components. It is known

Presented to the Society, November 25, 1950; received by the editors August 22, 1950.

${ }^{1} \mathrm{P}$. Dubreil, Quelques propriêtés des variêtês algêbriques se rattachant aux théories de l'algèbre moderne, Actualités Scientifiques et Industrielles, vol. 210, 1935. P. Dubreil, Sur la dimension des idéaux de polynomes, J. Math. Pures Appl. (9) vol. 15 (1936). These will be referred to as D1 and D2 respectively. 
(D1, Theorem II) that if $\mathfrak{a}$ is of the first species, and if $f(X)$ is a form which does not belong to any of the relevant prime ideals of $\mathfrak{a}$, then $\mathfrak{a}+R f$ has no irrelevant components. The ideal $\mathfrak{a}$ is said to be $h$ times of the first species if a sequence $L_{1}, L_{2}, \cdots, L_{h}$ of linear forms in $X_{0}, X_{1}, \cdots, X_{n}$ exists such that if $\mathfrak{a}_{0}=\mathfrak{a}, \mathfrak{a}_{i}=\mathfrak{a}+R L_{1}$ $+R L_{2}+\cdots+R L_{i}, i=1,2, \cdots, h$, then $L_{i}$ does not belong to any relevant prime ideal of $\mathfrak{a}_{i-1}$, and $\mathfrak{a}_{i}$ has no irrelevant components, $i=0,1, \cdots, h$. An algebraic variety $V$ in the $n$-dimensional projective space $P_{n}(k)$ is said to be $h$ times of the first species if $I(V)$, the ideal of $V$ in $R$, is $h$ times of the first species.

Let $V$ be an absolutely irreducible $r$-dimensional variety in $P_{n}(k)$, and let $\mathrm{D}=k\left[x_{0}, x_{1}, \cdots, x_{n}\right]$ be the ring generated by the homogeneous coordinates $\left(x_{0}, x_{1}, \cdots, x_{n}\right)$ of the general point of $V$. A set $\left(y_{0}, y_{1}, \cdots, y_{r}\right)$ of $r+1$ elements of $\mathrm{D}$ will be called an admissible set of independent variables for $D$ if (a) $y_{i}$ is homogeneous of degree one, and (b) $D$ is integrally dependent on $k\left[y_{0}, y_{1}, \cdots, y_{r}\right]$. The elements $y_{0}, y_{1}, \cdots, y_{r}$ will then be algebraically independent over $k$, and the quotients $y_{1} / y_{0}, y_{2} / y_{0}, \cdots, y_{r} / y_{0}$ will form a transcendence base for $\Sigma / k$, where $\Sigma$ is the field $k\left(x_{1} / x_{0}, x_{2} / x_{0}, \cdots, x_{n} / x_{0}\right)$ of rational functions on $V$.

Assume that $x_{0}, x_{1}, \cdots, x_{r}$ is an admissible set of independent variables for $\mathfrak{o}$, let $P=k\left(x_{1} / x_{0}, x_{2} / x_{0}, \cdots, x_{r} / x_{0}\right)$, and let $\nu$ be the relative degree $[\Sigma: P]$. Let $S=k\left[x_{0}, x_{1}, \cdots, x_{r}\right]$, and assume that there exist $\nu$ homogeneous elements, $\lambda_{1}, \lambda_{2}, \cdots, \lambda_{v}$, in 0 which are linearly independent over $S$ and which form a linear base for $\mathfrak{o}$ over $S$. It is known ${ }^{2}$ that if $\lambda_{i}$ is of degree of homogeneity $r_{i}$, and if $\omega$ is an element of 0 of degree of homogeneity $h$, then

$$
\omega=\sum_{i=1}^{\nu} P_{i}\left(x_{0}, x_{1}, \cdots, x_{r}\right) \lambda_{i},
$$

where $P_{i}$ is a form of degree $h-r_{i}$.

Lemma 1. If o possesses a linearly independent linear base $\lambda_{1}, \lambda_{2}, \cdots$, $\lambda_{\text {v }}$ over $S$, then $V$ is $r$ times of the first species.

Proof. Let $\mathfrak{a}=I(V)$, and let $T$ be the homomorphism $T f(X)$ $=f(x)$ of $R$ onto $\mathfrak{D}$ with kernel $\mathfrak{a}$. If $\mathfrak{a}_{i}=\mathfrak{a}+R X_{1}+R X_{2}+\cdots+R X_{i}$, then $T\left(\mathfrak{a}_{i}\right)=\mathfrak{b} x_{1}+b x_{2}+\cdots+b x_{i}$. We denote this ideal by $\mathfrak{m}_{i}$. Since $\mathbb{D}$ is integral over $S$, it follows that the ideal $\sum_{i=0}^{r} D x_{i}$ is an irrelevant ideal. Hence, after applying if necessary a suitable $k$-linear trans-

$2 \mathrm{H}$. T. Muhly, Independent integral bases and a characterization of regular surface, Trans. Amer. Math. Soc. vol. 54 (1943). The reference here is to the first part of the proof of Theorem 2.1. 
formation to $x_{0}, x_{1}, \cdots, x_{r}$ (and likewise to $X_{0}, X_{1}, \cdots, X_{r}$ ), we can assume that $X_{i}$ is not contained in any relevant prime ideal of $\mathfrak{a}_{i-1}$, and that $X_{0}$ does not belong to a relevant prime ideal of any of the ideals $\mathfrak{a}_{i}, i=1,2, \cdots, r$. Hence to show that $V$ is $r$ times of the first species it is necessary to show only that the ideal $\mathfrak{m}_{i}$ (and hence also $\mathfrak{a}_{i}$ ) has no irrelevant components.

Let $\mathrm{m}_{i}=A_{i} \cap Q_{i}$ where $A_{i}$ is the intersection of all of the primary ideals of $\mathfrak{m}_{i}$ which belong to relevant prime ideals, and $Q_{i}$ is a possible irrelevant component. If $Q_{i}$ is not redundant in the primary representation of $\mathfrak{m}_{i}$, then there exists an element $\eta \in A_{i}, \eta \notin Q_{i}$, such that $x_{0} \eta \in \mathfrak{m}_{i}$. If we write $\eta=\sum_{j=1}^{\nu} P_{j} \lambda_{j}, P_{j} \in S$, then since $\lambda_{1}, \lambda_{2}, \cdots, \lambda_{\nu}$ are independent over $S$, the coefficients $P_{1}, P_{2}, \cdots, P_{v}$ are uniquely determined by $\eta$. Now $x_{0} \eta=\sum\left(x_{0} P_{j}\right) \lambda_{j}=\omega_{1} x_{1}+\omega_{2} x_{2}+\cdots+\omega_{i} x_{i}$. On expressing the $\omega$ 's in terms of the $\lambda$ 's and comparing coefficients we find that $x_{0} P_{j} \in \mathfrak{m}_{i}, j=1,2, \cdots, \nu$. It follows that $x_{0} P_{j} \in\left(\mathfrak{m}_{i} \cap S\right)$, and since $\left(\mathfrak{m}_{i} \cap S\right)$ is the prime $S$-ideal, $S x_{1}+S x_{2}+\cdots+S x_{i}$, which does not divide $x_{0}$, we conclude that $P_{j} \in\left(\mathfrak{m}_{i} \cap S\right)$ and that $\eta \in \mathfrak{m}_{i}$. This is a contradiction, q.e.d.

3. To prove the converse of Lemma 1 we make use of Theorem II of D1. Let $V$ be an absolutely irreducible variety in $P_{n}(k)$ which is $r$ times of the first species. Use the same notations as in the preceding article, and assume that $x_{0}, x_{1}, \cdots, x_{r}$ is an admissible set of independent variables for $\boldsymbol{D}$.

LEмma 2. If $V$ is $r$ times of the first species, then there exist $\nu$ homogeneous elements $\lambda_{1}, \lambda_{2}, \cdots, \lambda_{v}$ in $\mathrm{o}$ which are linearly independent over $S$ and form a linear basis for o over $S$.

Proof. We can assume as in the proof of Lemma 1 that $X_{i}$ does not belong to any relevant component of $\mathfrak{a}_{i-1}$, and that $X_{0}$ does not belong to any relevant component of $a_{i}, i=1,2, \cdots, r$. Since $V$ is $r$ times of the first species the ideals $\mathfrak{a}_{1}, \mathfrak{a}_{2}, \cdots, \dot{\mathfrak{a}}_{r}$ have no irrelevant components (D1, Theorem II), whence the ideals $\mathfrak{m}_{1}, \mathfrak{m}_{2}, \cdots, \mathfrak{m}_{r}$ are likewise free of such components.

To construct the basis $\lambda_{1}, \lambda_{2}, \cdots, \lambda_{\nu}$ we proceed as follows. ${ }^{3}$ Let $\lambda_{1}$ be 1 , and when $\lambda_{1}, \cdots, \lambda_{i}$ have been defined, let $\lambda_{i+1}$ be a homogeneous element of $\mathfrak{D}$ of lowest degree $r_{i+1}$ such that $\lambda_{i+1}$ does not satisfy a congruence of the form

${ }^{3}$ This construction is discussed in detail for the case of algebraic surfaces in $\$ 8$ of the paper mentioned in footnote 2 . In the present note the construction is given in outline for the general case. 


$$
x_{0}^{k-r_{i+1}} \lambda_{i+1} \equiv \sum_{j=1}^{i} c_{j} x_{0}^{k-r j} \lambda_{j}\left(\mathfrak{m}_{r}\right), \quad \quad c_{j} \in k .
$$

Since the ideal $\mathrm{m}_{r}$ is generated by $x_{1}, x_{2}, \cdots, x_{r}$, it follows that its characteristic function $\chi\left(\mathfrak{m}_{r}, h\right)$ has the constant value $\nu$ if $h$ is sufficiently large, and since for any $h$ the functions $x_{0}^{h-r_{1}} \lambda_{1}, x_{0}^{h-r_{2}} \lambda_{2}, \cdots$, $x_{0}^{h-r_{i}} \lambda_{i}$ are $k$-linearly independent modulo $\mathrm{m}_{r}$, it follows that the above construction will terminate in exactly $\nu$ steps. If $\lambda_{1}, \lambda_{2}, \cdots, \lambda_{\nu}$ are the elements so obtained, and if $\omega$ is an element of 0 homogeneous of degree $h$, then for some integer $k, \omega$ will satisfy a congruence of the form

$$
x_{0}^{k-h} \omega \equiv \sum_{i=1}^{\nu} c_{i} x_{0}^{k-r i} \lambda_{i}\left(\mathfrak{m}_{r}\right)
$$$$
c_{i} \in k,
$$

whence

$$
x_{0}^{k-h}\left(\omega-\sum_{i=1}^{\nu} c_{i} x_{0}^{h-r_{i}} \lambda_{i}\right) \equiv 0\left(\mathfrak{m}_{r}\right) .
$$

Since $\mathfrak{m}_{r}$ has no irrelevant components and since $x_{0}$ is not divisible by any relevant prime ideal of $\mathrm{m}_{r}$, it follows that

$$
\omega=\sum_{i=1}^{\nu} c_{i} x_{0}^{h-r_{i}} \lambda_{i}+\sum_{j=1}^{r} \omega_{j} x_{j}
$$

where $\omega_{1}, \omega_{2}, \cdots, \omega_{r}$ are homogeneous elements of $\mathrm{D}$ of degree $h-1$. That $\lambda_{1}, \lambda_{2}, \cdots, \lambda_{\nu}$ form a basis for $\mathrm{D}$ over $S$ follows by induction on $h$ in view of equation (5). Since $\lambda_{1}, \lambda_{2}, \cdots, \lambda_{\nu}$ form a basis for $\mathfrak{D}$ over $S$ it follows that if $\bar{\lambda}_{i}=\lambda_{i} / x_{0}^{r_{i}}$, then $\bar{\lambda}_{1}, \bar{\lambda}_{2}, \cdots, \bar{\lambda}_{i}$ will form a linear basis for $\Sigma$ over $k\left(x_{1} / x_{0}, x_{2} / x_{0}, \cdots, x_{r} / x_{0}\right)$. Hence $\lambda_{1}, \lambda_{2}, \cdots$, $\lambda_{\nu}$ are linearly independent over $S$, q.e.d.

4. The criterion given in Lemmas 1 and 2 for the existence of an independent base for $\mathrm{o}$ over $S$ can be succinctly formulated in terms of the characteristic function of Hilbert, $\chi(\mathfrak{a}, h)$, associated with the variety $V .(\chi(\mathfrak{a}, h)$ is the number of forms of degree $h$ in $R$ which are linearly independent modulo $\mathfrak{a}=I(V)$.) Letting $\Delta$ denote the unit difference operator, $\Delta f(h)=f(h)-f(h-1)$, and using the same notation as above, we formulate the result as follows.

THEOREM. If $V$ is an absolutely irreducible $r$-dimensional variety in $P_{n}(k)$, then $a$ necessary and sufficient condition for the a-residues $x_{0}, x_{1}, \cdots, x_{r}$ of $X_{0}, X_{1}, \cdots, X_{r}$ to be an admissible set of independent variables for $\mathrm{D}$ and for $\mathrm{o}$ to possess an independent linear base 
over $S$ is that the characteristic function $\chi(a, h)$ satisfy the successive difference equations 4

$$
\Delta^{i} \chi(\mathfrak{a}, h)=\chi\left(\mathfrak{a}_{j}, h\right), \quad i=1,2, \cdots, r+1,
$$

for all values of $h$.

Proof. Since $\chi(a, h)$ is a polynomial of degree $r$ in $h$ when $h$ is sufficiently large, the last of equations (6) $(j=r+1)$ insures that for large values of $h, \chi\left(\mathfrak{a}_{r+1}, h\right)=0$. This means that $\mathfrak{a}_{r+1}$ is projectively irrelevant, and by a theorem of Zariski ${ }^{5}$ this will be so if and only if $x_{0}, x_{1}, \cdots, x_{r}$ form an admissible set of independent variables for D. A straightforward computation yields the conclusion that equations (6) are valid for all $h$ if and only if the equations

$$
\Delta \chi\left(\mathfrak{a}_{i}, h\right)=\chi\left(\mathfrak{a}_{i+1}, h\right), \quad i=0,1, \cdots, r,
$$

are likewise valid for all $h$. By Theorem I of D1, this will be so if and only if the ideals $\mathfrak{a}_{1}, \mathfrak{a}_{2}, \cdots, \mathfrak{a}_{r}$ are free of irrelevant components, or if and only if $V$ is $r$ times of the first species. The theorem therefore follows from Lemmas 1 and 2, q.e.d.

\section{State University of Iowa}

4 The ideal $a_{r+1}$ is defined to be $a+R X_{0}+R X_{1}+\cdots+R X_{r}$. It should be pointed out that the $x$ function of any ideal is defined to be zero for negative values of the argument $h$.

- O. Zariski, Foundations of a general theory of birational correspondences, Trans. Amer. Math. Soc. vol. 53 (1943). The reference here is to part II, \$2. 